\title{
Phototherapy with turquoise versus blue light
}

\author{
F Ebbesen, G Agati, R Pratesi
}

Arch Dis Child Fetal Neonatal Ed 2003;88:F430-F43 1

Preterm jaundiced infants were treated by phototherapy with a new turquoise fluorescent lamp. This was more effective in reducing plasma total bilirubin in relation to light irradiance than the ubiquitously used blue fluorescent lamp.

$\mathrm{P}$ hototherapy is the most widespread treatment for lowering bilirubin concentration in neonates. Blue fluorescent lamps with emission peak wavelength matching the absorption maximum of the plasma bilirubin-albumin complex at $460 \mathrm{~nm}$ have been used successfully in phototherapy, in particular the Philips F20WT12/BB special blue lamp with peak emission at $446 \mathrm{~nm}$ and the Philips 20WTL/52 blue lamp with peak emission at $452 \mathrm{~nm}$ and spectral width (width at half maximum) of $55 \mathrm{~nm}$.

An increase in phototherapeutic efficiency was predicted for lamps with emission peak at about $490 \mathrm{~nm} .^{1}$ Thus, nonstandard, cold cathode fluorescent lamps emitting at $490 \mathrm{~nm}$ were developed and clinically tested with successful results. ${ }^{2}$ Then new turquoise (blue-green) standard fluorescent lamps were made by Osram, with emission peak at $490 \mathrm{~nm}$ and bandwidth of $65 \mathrm{~nm}$.

In this study, the phototherapeutic efficiencies of the turquoise and Philips/52 blue lamps are compared.

\section{METHODS}

The study period was 1 November 2000 to 31 December 2001. The inclusion criteria were preterm infants with a gestational age of 196-258 days, a postnatal age of $>24$ hours, and nonhaemolytic hyperbilirubinaemia. The infants participated in the study for 48 hours.

Phototherapy was administered continuously, except during feeding, nursing care, and blood sampling. Treatment took place in either an incubator or a bassinet. The distance from the phototherapy apparatus to the surface of the infants was about $32 \mathrm{~cm}$.

The infants were randomised to one of two regimens: six of the turquoise fluorescent lamps $(18 \mathrm{~W})$ plus two daylight fluorescent lamps (Philips TL-D18W/96) to reduce inconvenience caused to staff; or six blue fluorescent lamps (Philips 20WTL/ 52) plus two of the daylight lamps. The geometries of the two lamp systems were identical. The lamps were kept on for 100 hours before being used for phototherapy, and replaced after 1000 hours of operation. In fig $1 \mathrm{~A}$, the calibrated emission spectra of a single turquoise and blue lamp is shown along with the absorption spectrum of $(\mathrm{Z}, \mathrm{Z})$-bilirubin $(20 \mu \mathrm{mol} / \mathrm{l})$, in $0.1 \mathrm{~mol} / \mathrm{l}$ sodium phosphate buffer, $\mathrm{pH} 7.4$, containing human serum albumin $(40 \mu \mathrm{mol} / \mathrm{l})$, normalised to its maximum value.

The light irradiance of the two phototherapy units was measured daily on the front/back of the infant's chest with a broad band photodiode power meter (EG\&G, model 460; Salem, Massachusetts, USA), previously calibrated at the two emission bands, 452 and $490 \mathrm{~nm}$, against a thermopile (Nova power meter, 2A thermal head; Ophir Optronics Ltd, Jerusalem, Israel)
Total serum bilirubin concentration was measured with a Vitros 950 analyser on capillary blood drawn by heel prick 0 , 12, 24, and 48 hours after the start of phototherapy. The imprecision expressed as a coefficient of variation was $1.9 \%$ at a bilirubin level of $225 \mu \mathrm{mol} / \mathrm{l}$.

A questionnaire on inconvenience caused by working in the turquoise or blue light was completed by 47 staff members. Problems included irritation to the eyes, temporary glare, temporary blurred vision, headache, nausea, giddiness, and difficulty in observing the infant.

The study was approved by the ethics committee for North Jutland County. Written informed consent was obtained from the parents.

\section{RESULTS AND DISCUSSION}

The characteristics of the infants treated with turquoise light $(\mathrm{n}=42)$ compared with those of the infants treated with blue light $(\mathrm{n}=43)$ were: birth weight $(\mathrm{g})$ (median (range)), 1853 (951-3225) v 1940 (845-3550); gestational age (days) (median (range)), 229 (198-258) v 235 (198-255); sex female/male (number), 17:25 v 19:24; respiratory distress syndrome (number), $13 \vee 10$; infants suspected of infection (number), 3 v 2; incubator/bassinet (number), 28:14 v 29:14; age at start of phototherapy (hours) (median (range)), 74 $(25-234) v 69$ (37-260); percentage of study period in phototherapy (median (range)), 86 (68-100) v 82 (69-100).

Figure $1 \mathrm{~B}, \mathrm{C}$ shows the decrease in total serum bilirubin concentration during phototherapy. No significant difference in the decrease after 48 hours of treatment was found between infants treated with turquoise light and blue light $(p=0.36$; Mann-Whitney U test).

No side effects were observed except for loose green stools.

Light irradiance values (mean (SD)) for the turquoise, blue, and daylight lamps at the body surface were $2.72(0.25), 3.52$ (0.33), and $0.56(0.07) \mathrm{mW} / \mathrm{cm}^{2}$ respectively.

The fall in light intensity over 1000 hours of operation was similar for the turquoise and blue lamps (11\% and $14 \%$ respectively).

As the light irradiance of the treatment with turquoise light was about three quarters of that with blue light and the reduction in plasma bilirubin concentration was the same in the two groups, the phototherapeutic efficiency of the new lamps is considerably higher $(30 \%)$. This is consistent with the most efficient spectral emission predicted $^{1}$ and confirms previous preliminary results. $^{2}$

There were no significant differences in the inconvenience to staff members between turquoise and blue light ( $p>0.05$; $\chi^{2}$ test). Most had no preference for either type of light. If they had a preference, there was no significant difference between the turquoise and blue light.

The results of our investigation indicate that the new Osram turquoise fluorescent lamps and the Philips/52 blue lamps are equally convenient to use for the treatment of jaundiced neonates. However, the turquoise lamps are preferable because of the more efficient reduction in plasma bilirubin concentration in relation to light irradiance and the less severe side effects, such as mutagenic ${ }^{3}$ and photodegradation processes, ${ }^{4}$ 

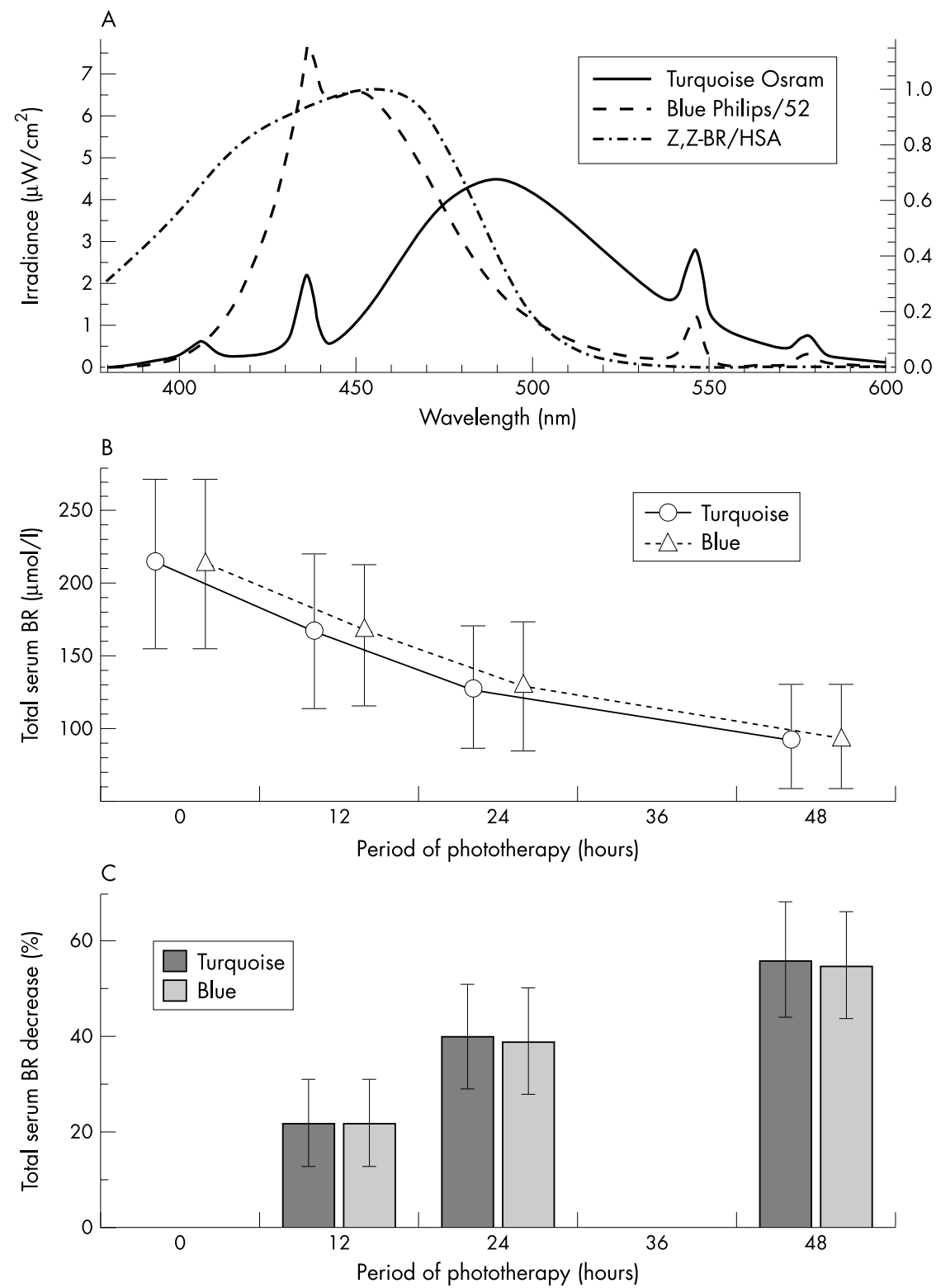

Figure 1 (A) Emission spectra of a single turquoise (Osram $18 \mathrm{~W}$ ) and blue (Philips 20WTL/52) tube

2 measured at the same distance (30

$\varangle \mathrm{cm}$ ) with a calibrated

o spectroradiometer. The irradiance

믈 values of the tubes can be compared.

Narrow peaks appearing on the

O broad fluorescence bands are due to

$\varangle$ the mercury emission lines. The

T) absorption spectrum of $(Z, Z)$-bilirubin

(BR)/human serum albumin (HSA) is

Nे also shown. AU, Arbitrary units. (B)

N Decrease in total serum BR at 12,24,

and 48 hours after the start of

phototherapy for the turquoise and blue light. (C) Perceptual decrease in total serum BR at 12,24, and 48

hours after the start of phototherapy.

Values in $(B)$ and $(C)$ are mean $(S D)$. associated with light of longer wavelength. Further improvement is expected from the introduction of lamp phosphor with narrower spectral emission in the blue-green spectral region.

\section{ACKNOWLEDGEMENTS}

We thank Osram, especially Dr M Zachau, for providing the turquoise lamps.

\section{Authors' affiliations}

F Ebbesen, Department of Pediatrics, University Hospital of Aalborg, Denmark

G Agati, Institute of Applied Physics-CNR, Sezione INFM di Firenze, Florence, Italy

R Pratesi, Dipartimento di Fisica, Università di Firenze, Sezione INFM di Firenze
Correspondence to: Dr Ebbesen, Department of Pediatrics, Aalborg University Hospital, DK 9000 Aalborg, Denmark; u19091@aas.nja.dk

Accepted 1 October 2002

\section{REFERENCES}

1 Agati G, Fusi F, Donzelli GP, et al. Quantum yield and skin filtering effects on the formation rate of lumirubin. J Photochem Photobiol $B$ 1993; 18:197-203.

2 Donzelli GP, Pratesi S, Rapisardi G, et al. 1-day phototherapy of neonatal jaundice with blue-green lamp. Lancet 1995;346:184-5.

3 Rosenstein BS, Ducore JM. Induction of DNA strand breaks in normal human fibroblasts exposed to monochromatic ultraviolet and visible wavelengths in the 240-546 nm range. Photochem Photobiol 1983;38:51-5.

4 Rudolph N, Parekh AJ, Hittelman J, et al. Postnatal decline in pyridoxal phosphate and riboflavin. Accentuation by phototherapy. American Journal of Diseases in Children 1985;139:812-15. 